\title{
Development of MRC Centre MRI calf muscle fat fraction protocol as a sensitive outcome measure in Hereditary Sensory Neuropathy Type 1
}

Kugathasan, Umaiyal ; Evans, Matthew R B ; Morrow, Jasper M ; Sinclair, Christopher D J ; Thornton, John S ; Yousry, Tarek A ; Hornemann, Thorsten ; Suriyanarayanan, Saranya ; Owusu-Ansah, Khadijah

; Lauria, Giuseppe ; Lombardi, Raffaella ; Polke, James M ; Wilson, Emma ; Bennett, David L H ; Houlden, Henry ; Hanna, Michael G ; Blake, Julian C ; Laura, Matilde ; Reilly, Mary M

\begin{abstract}
OBJECTIVES Hereditary sensory neuropathy type 1 (HSN1) is a rare, slowly progressive neuropathy causing profound sensory deficits and often severe motor loss. L-serine supplementation is a possible candidate therapy but the lack of responsive outcome measures is a barrier for undertaking clinical trials in HSN1. We performed a 12-month natural history study to characterise the phenotype of HSN1 and to identify responsive outcome measures. METHODS Assessments included Charcot-MarieTooth Neuropathy Score version 2 (CMTNSv2), CMTNSv2-Rasch modified, nerve conduction studies, quantitative sensory testing, intraepidermal nerve fibre density (thigh), computerised myometry (lower limbs), plasma 1-deoxysphingolipid levels, calf-level intramuscular fat accumulation by MRI and patientbased questionnaires (Neuropathic Pain Symptom Inventory and 36-Short Form Health Survey version 2 [SF-36v2]). RESULTS 35 patients with HSN1 were recruited. There was marked heterogeneity in the phenotype mainly due to differences between the sexes: males generally more severely affected. The outcome measures that significantly changed over 1 year and correlated with CMTNSv2, SF-36v2-physical component and disease duration were MRI determined calf intramuscular fat accumulation (mean change in overall calf fat fraction $2.36 \%, 95 \%$ CI 1.16 to $3.55, \mathrm{p}=0.0004$ ), pressure pain threshold on the hand (mean change $40 \mathrm{kPa}, 95 \%$ CI 0.7 to $80, \mathrm{p}=0.046$ ) and myometric measurements of ankle plantar flexion (median change $-0.5 \mathrm{Nm}, \mathrm{IQR}-9.5$ to $0, \mathrm{p}=0.0007$ ), ankle inversion (mean change $-0.89 \mathrm{Nm}, 95 \% \mathrm{CI}-1.66$ to $-0.12, \mathrm{p}=0.03$ ) and eversion (mean change $-1.61 \mathrm{Nm}, 95 \% \mathrm{CI}-2.72$ to $-0.51, \mathrm{p}=0.006$ ). Intramuscular calf fat fraction was the most responsive outcome measure. CONCLUSION MRI determined calf muscle fat fraction shows validity and high responsiveness over 12 months and will be useful in HSN1 clinical trials.
\end{abstract}

DOI: https://doi.org/10.1136/jnnp-2018-320198

Posted at the Zurich Open Repository and Archive, University of Zurich

ZORA URL: https://doi.org/10.5167/uzh-171620

Journal Article

Published Version

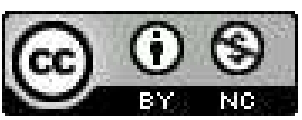

The following work is licensed under a Creative Commons: Attribution-NonCommercial 4.0 International (CC BY-NC 4.0) License. 
Originally published at:

Kugathasan, Umaiyal; Evans, Matthew R B; Morrow, Jasper M; Sinclair, Christopher D J; Thornton, John S; Yousry, Tarek A; Hornemann, Thorsten; Suriyanarayanan, Saranya; Owusu-Ansah, Khadijah; Lauria, Giuseppe; Lombardi, Raffaella; Polke, James M; Wilson, Emma; Bennett, David L H; Houlden, Henry; Hanna, Michael G; Blake, Julian C; Laura, Matilde; Reilly, Mary M (2019). Development of MRC Centre MRI calf muscle fat fraction protocol as a sensitive outcome measure in Hereditary Sensory Neuropathy Type 1. Journal of Neurology, Neurosurgery, and Psychiatry, 90(8):895-906.

DOI: https://doi.org/10.1136/jnnp-2018-320198 


\title{
Development of MRC Centre MRI calf muscle fat fraction protocol as a sensitive outcome measure in Hereditary Sensory Neuropathy Type 1
}

\author{
Umaiyal Kugathasan, ${ }^{1}$ Matthew R B Evans, ${ }_{1}^{1,2}$ Jasper M Morrow, ${ }^{1,2}$ \\ Christopher D J Sinclair, ${ }^{1,2}$ John S Thornton, ${ }^{1,2}$ Tarek A Yousry, ${ }^{1,2}$ Thorsten Hornemann, ${ }^{3}$ \\ Saranya Suriyanarayanan, ${ }^{3}$ Khadijah Owusu-Ansah, ${ }_{1}^{4}$ Giuseppe Lauria, ${ }^{5,6}$ \\ Raffaella Lombardi, ${ }^{5}$ James M Polke, ${ }^{7}$ Emma Wilson, ${ }_{1}^{1}$ David L H Bennett, ${ }^{8}$ \\ Henry Houlden, ${ }^{1}$ Michael G Hanna, ${ }^{1}$ Julian C Blake, ${ }^{1,9}$ Matilde Laura, ${ }^{1}$ Mary M Reilly ${ }^{1}$
}

For numbered affiliations see end of article.

\section{Correspondence to} Professor Mary M Reilly, MRC Centre for Neuromuscular Diseases, UCL Institute of Neurology and National Hospital for Neurology and Neurosurgery, London WC1N 3BG, UK; m.reilly@ucl.ac.uk

Received 13 December 2018 Revised 19 February 2019 Accepted 21 February 2019 Published Online First 17 April 2019

Check for updates

(C) Author(s) (or their employer(s)) 2019. No commercial re-use. See rights and permissions. Published by BMJ.

To cite: Kugathasan U, Evans MRB, Morrow JM, et al. J Neurol Neurosurg Psychiatry 2019:90:895-906.

\section{ABSTRACT}

Objectives Hereditary sensory neuropathy type 1

(HSN1) is a rare, slowly progressive neuropathy causing profound sensory deficits and often severe motor loss.

L-serine supplementation is a possible candidate therapy but the lack of responsive outcome measures is a barrier for undertaking clinical trials in HSN1. We performed a 12-month natural history study to characterise the phenotype of HSN1 and to identify responsive outcome measures.

Methods Assessments included Charcot-Marie-Tooth Neuropathy Score version 2 (CMTNSv2), CMTNSv2Rasch modified, nerve conduction studies, quantitative sensory testing, intraepidermal nerve fibre density (thigh), computerised myometry (lower limbs), plasma 1-deoxysphingolipid levels, calf-level intramuscular fat accumulation by MRI and patient-based questionnaires (Neuropathic Pain Symptom Inventory and 36-Short Form Health Survey version 2 [SF-36v2]).

Results 35 patients with HSN1 were recruited. There was marked heterogeneity in the phenotype mainly due to differences between the sexes: males generally more severely affected. The outcome measures that significantly changed over 1 year and correlated with CMTNSv2, SF-36v2-physical component and disease duration were MRI determined calf intramuscular fat accumulation (mean change in overall calf fat fraction $2.36 \%, 95 \% \mathrm{Cl} 1.16$ to $3.55, \mathrm{p}=0.0004$ ), pressure pain threshold on the hand (mean change $40 \mathrm{kPa}, 95 \% \mathrm{Cl} 0.7$ to $80, p=0.046$ ) and myometric measurements of ankle plantar flexion (median change $-0.5 \mathrm{Nm}, \mathrm{IQR}-9.5$ to $0, p=0.0007$ ), ankle inversion (mean change $-0.89 \mathrm{Nm}$, $95 \% \mathrm{Cl}-1.66$ to $-0.12, \mathrm{p}=0.03$ ) and eversion (mean change $-1.61 \mathrm{Nm}, 95 \% \mathrm{Cl}-2.72$ to $-0.51, \mathrm{p}=0.006$ ). Intramuscular calf fat fraction was the most responsive outcome measure.

Conclusion MRI determined calf muscle fat fraction shows validity and high responsiveness over 12 months and will be useful in HSN1 clinical trials.

\section{INTRODUCTION}

Hereditary sensory neuropathy type 1 (HSN1), due to SPTCL1 and SPTLC2 gene mutations, is an autosomal dominant sensory predominant neuropathy. ${ }^{1}$ Painless slowly healing ulcers, which can lead to osteomyelitis and amputations, are frequent. Motor involvement is variable ${ }^{1}$ and neuropathic pain is common. ${ }^{2}$ As a result of a founder mutation in the UK, SPTLC1 (C133W) is the most common mutation. ${ }^{3}$

SPTLC1 and SPTLC2 encode for two subunits of the enzyme serine palmitoyltransferase (SPT) which catalyses the rate-limiting step in de novo sphingolipid biosynthesis. SPTLC1 and SPTLC2 mutations alter the substrate specificity resulting in the production of 1-deoxysphingolipids (1-deoxySL) which have been shown to be neurotoxic in chick primary dorsal root ganglion and motor neuron cultures. ${ }^{4}$ Both transgenic mice expressing mutant SPTLC1 and patients with HSN1 have elevated plasma levels of 1-deoxySLs. ${ }^{45}$ A study using transgenic mice expressing mutant SPTLC1 has demonstrated a mild beneficial treatment effect with oral L-serine. ${ }^{6}$ In the same study, a 10 -week pilot trial of L-serine in patients with HSN1 showed that L-serine was well tolerated and led to a reduction in plasma 1-deoxySL levels.

A small (18 patients) randomised placebo-controlled trial in HSN1 which has just been published has shown that oral L-serine treatment potentially slows disease progression. ${ }^{7}$ A larger definitive efficacy trial of serine in the HSN1 population is warranted, however the major barrier is the lack of natural history data and responsive outcome measures.

Difficulties in conducting clinical trials due to the lack of responsive outcome measures in slowly progressive inherited neuropathies have been highlighted in ascorbic acid trials in Charcot-Marie-Tooth disease $1 \mathrm{~A}$ (CMT1A) ${ }^{8-12}$ In addition to failing to detect any benefit with ascorbic acid, the studies demonstrated no significant changes in their primary outcome measures over 2 years, and negligible to small responsiveness of the secondary outcome measures.

We recently demonstrated that MRI quantification of calf intramuscular fat shows high responsiveness over 12 months in patients with CMT1A. ${ }^{13}$ These difficulties are compounded in a rare condition like HSN1 where limited patient numbers and phenotypic heterogeneity require even more responsive outcome measures. 
To address the lack of natural history data and to develop responsive outcome measures, we performed a prospective natural history study to extensively characterise the HSN1phenotype and identify responsive markers of disease progression over 1 year using a variety of tests including MRI.

\section{MATERIALS AND METHODS}

Patients over the age of 18 with genetically confirmed HSN1 due to SPTLC1 and SPTLC2 mutations were identified from the Neurogenetics Laboratory, National Hospital for Neurology and Neurosurgery and Bristol Genetics Laboratory (online supplementary figure 1). Exclusion criteria were concomitant neuromuscular disease, significant comorbidities including medication-dependent diabetes and safety-related MRI contraindications. Age and sex-matched control subjects $(50 \%$ males, mean age of males $=44.4$ years [SD: 7.7, range 36-51], mean age of females $=41.6$ years [SD: 12.6, range 24-57]) were enrolled for the MRI (previously validated) ${ }^{13}$ part of the study to ensure any changes noted in the patients were related to disease rather than to MRI/technical factors. The healthy controls (no history of neuromuscular disorders) were recruited from departmental staff and their families.

\section{Study design}

Patients were assessed at baseline and after 12 months. At the baseline visit, demographic and clinical information was collected in a standardised form. The following assessments were performed by a single researcher (UK): Charcot-Marie-Tooth Neuropathy Score version 2 (CMTNSv2), ${ }^{14}$ nerve conduction studies (NCS), quantitative sensory testing (QST), computerised myometry and skin biopsies. The MRI data were analysed by a single researcher (MRBE) who was blinded to diagnosis.

\section{Assessments}

Clinical impairment was assessed using the CMTNSv2 $2,{ }^{14}$ a composite score (0-36 points) of patient symptoms, examination findings and neurophysiological assessment. The more recent Rasch-modified CMTNSv2 (CMTNSv2-R) ${ }^{15}$ was also used, however since it did not have greater sensitivity in picking up change, CMTNSv2 measurements are quoted in the results.

NCS were performed using standard techniques. ${ }^{16}$ Measurements from the right and left sides were averaged to give an overall value for each nerve.

QST was performed according to the published protocol of the German Research Network on Neuropathic Pain. ${ }^{17}$ The following parameters were tested: cold detection threshold (CDT), warm detection threshold (WDT), thermal sensory limen (TSL), cold pain threshold (CPT), heat pain threshold (HPT), mechanical detection threshold (MDT), mechanical pain threshold (MPT), vibration detection threshold (VDT) and pressure pain threshold (PPT). Paradoxical heat sensation (PHS), mechanical pain sensitivity (MPS), dynamic mechanical allodynia (DMA) and wind-up ratio (WUR) were also assessed at baseline. QST was also performed on the left trunk area when undetectable thermal thresholds were noted in the hands. ${ }^{18}$ For baseline measures, Z-scores were calculated $[Z$-score $=$ (value of patient - mean value of controls)/SD of controls]. ${ }^{17}$

Bilateral lower limb myometry was performed using HUMAC NORM dynamometer (CSMi, MA, USA). The protocol ${ }^{13}$ included isometric measurements of knee extension and flexion, ankle dorsiflexion and plantar flexion, and ankle inversion and eversion. Measurements for the right and left sides were averaged.
A $3 \mathrm{~mm}$ diameter skin punch biopsy was taken on the lateral side of the thigh. This site rather than the conventional site of distal leg was chosen in accordance with ethical advice due to concerns over poor healing and floor effects in patients with this length-dependent neuropathy. Specimen processing and quantification of the intraepidermal nerve fibre densities (IENFD) were performed in the 'Carlo Besta' Neurological Institute, Milan, Italy, according to established guidelines. ${ }^{19}$

Plasma 1-deoxySL levels were analysed at the Institute of Clinical Chemistry, University Hospital, Zurich, Switzerland. 1-deoxySL (1-deoxysphinganine and 1-deoxysphingosine) levels were measured as already described ${ }^{20}$ and compared with laboratory controls. Both baseline and follow-up samples were analysed together to eliminate interassay variability and were calibrated using internal standards.

The Neuropathic Pain Symptom Inventory (NPSI) and SF-36 (version 2$)^{21}$ were used to assess pain and quality of life, respectively. Norm-based scoring was used for SF-36 where lower scores reflect poorer health status. ${ }^{22}$

For the MRI, both lower limbs were examined in a 3T MRI scanner (TIM Trio, Siemens, Erlangen, Germany). As part of a wider protocol, the three-point Dixon technique ${ }^{23}$ was used for fat quantification of the proximal calf muscles with $82 \times 5 \mathrm{~mm}$ contiguous slices, $512 \times 240$ matrix. Individual muscle-group regions of interest (ROI) were manually defined by a single observer. Bilateral ROIs were defined for seven lower limb muscles at a level of $130 \mathrm{~mm}$ distal to the tibial plateau: tibialis anterior, extensor hallucis longus, peroneus longus, medial gastrocnemius, lateral gastrocnemius, soleus and the deep posterior group. For all individual ROI, custom-written software extracted individual muscle-group ROI mean fat fraction (\%) and cross-sectional area in millimeter squared. Full details of acquisition protocol and MRI analysis methods are found in the online supplementary method 1 .

Right and left leg MRI fat fraction values for each muscle were averaged to give an overall mean value per patient. A summary measure ('overall calf muscle fat fraction') was calculated for each participant, representing the mean combined fat fraction of all ROI across both lower limbs weighted to cross-sectional surface area.

\section{Statistical analysis}

SPSS statistics V.22 (IBM) and GraphPad Prism V.6 (GraphPad Software, La Jolla, California, USA) were used for statistical analysis. All data were tested for normality by Shapiro-Wilk test in conjunction with visual inspection of frequency distribution graphs. For cross-sectional baseline data, mean values/SDs and median/IQR are given as appropriate. Mann-Whitney test was used for comparison between controls and patients for the MRI fat fraction measurements.

Change over 1 year (follow-up minus baseline) was represented as means/95\% CI or medians/IQR (as appropriate). Missing data were excluded from the analysis and change was only calculated in patients who had both baseline and 12-month follow-up data. The significance of mean change was evaluated using one-sample two-tailed t-tests (normally distributed) and one-sample Wilcoxon signed-rank tests (non-normally distributed). Statistical significance was set at 5\%.

Linear regression was performed to assess the dependence of CMTNSv2 on gender and disease duration. Spearman's rank correlation was used to assess correlation between CMTNSv2, SF-36v2, disease duration and other tests performed in the study. Correlation with plasma 1-deoxySL levels was assessed 
Table 1 Baseline characteristics:patient demographics and clinical history

\begin{tabular}{|c|c|c|c|c|}
\hline & \multicolumn{2}{|l|}{ SPTLC1 } & \multicolumn{2}{|l|}{ SPTLC2 } \\
\hline & Males & Females & Males & Females \\
\hline Patients & 18 & 13 & 2 & 2 \\
\hline Mutation & C133W & C133W & S384F & $\begin{array}{l}\text { S384F } \\
\text { A182P }\end{array}$ \\
\hline $\begin{array}{l}\text { Mean age, years (SD; } \\
\text { range) }\end{array}$ & $\begin{array}{l}48.9(13.1 ; \\
23-71)\end{array}$ & $\begin{array}{l}43.9 \\
(17.3 ; \\
20-73)\end{array}$ & $52.5(49-56)$ & $66.5(65-68)$ \\
\hline $\begin{array}{l}\text { Mean disease } \\
\text { duration, years } \\
\text { (SD; range) }\end{array}$ & $\begin{array}{l}27.2(14.1 ; \\
7-54)\end{array}$ & $\begin{array}{l}18.8 \\
(15.6 \\
3-63)\end{array}$ & $16(16-16)$ & $36.5(21-52)$ \\
\hline $\begin{array}{l}\text { Mean age at onset, } \\
\text { years } \\
\text { (SD; range) }\end{array}$ & $\begin{array}{l}21.7(7.2 \\
12-37)\end{array}$ & $\begin{array}{l}25.1 \\
(14.3 \\
10-50)\end{array}$ & $36.5(33-40)$ & $30.0(13-47)$ \\
\hline $\begin{array}{l}\text { Mean age at first } \\
\text { ulcer, years } \\
\text { (SD; range) }\end{array}$ & $\begin{array}{l}28.0(10.9 ; \\
16-63)\end{array}$ & $\begin{array}{l}28.6 \\
(15.8 \\
10-65) \\
(n=12)\end{array}$ & $\begin{array}{l}41 \\
(n=1)\end{array}$ & $31.5(13-50)$ \\
\hline $\begin{array}{l}\text { Mean age at onset } \\
\text { of neuropathic pain, } \\
\text { years (SD; range) }\end{array}$ & $\begin{array}{l}30.6(12.9 ; \\
14-61) \\
(n=16)\end{array}$ & $\begin{array}{l}30.8 \\
(13.4 \\
15-52) \\
(n=12)\end{array}$ & $40.5(40-41)$ & $54.0(53-55)$ \\
\hline $\begin{array}{l}\text { Complication: } \\
\text { osteomyelitis }\end{array}$ & $33.3 \%(6 / 18)$ & $0 \%$ & $50.0 \%(1 / 2)$ & $0 \%$ \\
\hline $\begin{array}{l}\text { Complication: } \\
\text { amputation }\end{array}$ & $5.6 \%(1 / 18)$ & $0 \%$ & $50.0 \%(1 / 2)$ & $50.0 \%(1 / 2)$ \\
\hline $\begin{array}{l}\text { CMTNSv2 } \\
\text { (SD; range) }\end{array}$ & $\begin{array}{l}26.8(5.1 ; \\
16-35) \\
(n=17)\end{array}$ & $\begin{array}{l}14.2 \\
(10.4 ; \\
3-24)\end{array}$ & $13.5(5-22)$ & $20.0(15-25)$ \\
\hline $\begin{array}{l}\text { CMTNSv2-R } \\
\text { (SD; range) }\end{array}$ & $\begin{array}{l}31.5(5.9 ; \\
17-39)\end{array}$ & $\begin{array}{l}16.5 \\
(12.1 ; \\
4-39)\end{array}$ & $15.0(6-24)$ & $24.0(19-29)$ \\
\hline
\end{tabular}

$\mathrm{n}$ is the number of patients reporting the specified symptom when different from that quoted at the top of the table.

CMTNSv2, Charcot-Marie-Tooth Neuropathy Score version 2; CMTNSv2-R, CMTNSv2-Rasch modified.

following normalisation of plasma 1-deoxySL by log transformation. Statistical significance was set at $0.1 \%(p<0.001)$ following Bonferroni correction for multiple comparisons.

The standardised response mean (SRM), ${ }^{24}$ also known as Cohen's d, was used to measure effect size. SRM is an established term in this field as a measure of outcome responsiveness and will enable comparison between different outcome measures within and between studies. ${ }^{1325}$ It was calculated by dividing the mean change (follow-up - baseline) by the SD. SRMs of 0.20 $0.49,0.50-0.79$ and $\geq 0.80$ reflect small, moderate and large responsiveness, respectively. ${ }^{25}$

\section{RESULTS}

Between September 2013 and July 2014, a total of 35 patients and 10 healthy MRI controls were recruited. The demographic and clinical baseline profiles are presented in table 1 and figure 1. There was no significant difference in age at baseline between males and females.

Loss of sensation (43\% of patients) followed by painless foot injury (31\% of patients) were the most common initial symptoms. A greater heterogeneity was seen in SPTLC1 females compared with the SPTLC1 males with a larger variation in age of onset (range 10-50 years vs 12-37 years [figure 1A]) and disease severity (CMTNSv2 range $=3-34$ vs $16-35$ [figure $1 \mathrm{~B}, \mathrm{C}$ ]). There was also a variation in disease progression within SPTLC1 females. There was a significant difference $(p=0.002)$ in disease severity as determined by the CMTNSv2 between the males and females, with males being more severely affected (figure 1C). In patients with SPTLC1 $(\mathrm{C} 133 \mathrm{~W})$ mutations, gender was a significant predictor of CMTNSv2 $\left(\mathrm{R}^{2}=0.38, \beta=-0.63, \mathrm{p}<0.001\right)$ accounting for $40 \%$ of the variation seen in CMTNSv2. Disease duration accounted for $46 \%$ of the variation in CMTNSv2 $\left(\mathrm{R}^{2}=0.44, ß=0.68, \mathrm{p}<0.001\right)$.

NCS and QST showed a clear length-dependent pattern of sensory and motor deficits (table 2, figure 1D-J). QST profiles illustrate early small (CDT, WDT) and large fibre (MDT, VDT) involvement in HSN1. All the QST parameters showed predominantly a loss of function (negative Z-scores); however, PPT was reduced (gain of function) in a subset of patients when tested at the hand, trunk and face. The distribution of the abnormal PPTs (face $>$ trunk $>$ hands) suggests there might be early hypersensitivity followed by decreased sensitivity as the neuropathy progresses. A small number of patients were hyperalgesic with increased MPS in the hands (6\% [2/35]) and trunk (15\% [3/20]). None of the patients had allodynia (determined by DMA) or increased temporal summation of pain (determined by WUR).

Results of the computerised myometry are summarised in table 3. Ankle dorsiflexion was the worst affected with zero power detected in $68 \%(13 / 19)$ and $20 \%(3 / 15)$ of male/female patients.

Skin biopsy analysis showed complete denervation in $60 \%$ $(12 / 20)$ of male patients and 21\% (3/14) of female patients (figure $2 \mathrm{~A}$ and table 3 ). All patients had severely reduced IENFD (median $0.1[\mathrm{IQR}=0.0-1.8]$ ) compared with published normative data ${ }^{26}$ apart from two patients with the mildest phenotype (CMTNSv2 $\leq 5)$. Plasma 1-deoxysphinganine levels were elevated in all patients compared with laboratory controls. There were no significant differences in the plasma 1-deoxysphinganine $(p=0.17)$ levels between males and females (figure $2 \mathrm{~B}$ and table 3 ). In males, plasma 1-deoxysphinganine level correlated moderately $\left(r_{s}=0.549, \mathrm{p}=0.01\right)$ with CMTNSv2 whereas there was no correlation between plasma 1-deoxysphinaganine $\left(r_{s}=0.274, \mathrm{p}=0.32\right)$ level and CMTNSv2 (figure 2C) in females.

The pain profile was similar between males and females (figure 2D) with no significant difference $(p=0.65)$ in the total NPSI score (table 3 ). All but one patient had the typical lancinating pain. There was no correlation between total NPSI score and plasma 1-deoxysphinganine level $(r=-0.02)$. In the SF-36v2 for the whole cohort, physical domains had lower scores compared with age-matched controls (table 3).

For the MRI assessments, 34 patients with HSN and 10 controls were analysed at baseline and the results are summarised in table 3. Representative images are shown in figure $2 \mathrm{E}$. The combined overall muscle and each individual muscle mean fat fractions were significantly higher in patients with HSN than healthy controls for the whole cohort ( $p$ values range $=<0.001$ ) and when subdivided into gender ( $\mathrm{p}$ values range $=0.001$ to $<0.001$ in males, 0.02 to $<0.001$ in females). Overall calf muscle fat fraction correlated strongly with CMTNSv2 in both males $\left(r_{\mathrm{s}}=0.850, \mathrm{p}<0.0001\right)$ and females $\left(r_{\mathrm{s}}=0.859, \mathrm{p}<0.0001\right)$ (figure $2 \mathrm{~F}$ ). Once there is motor involvement, intramuscular fat accumulation progresses similarly between males and females (figure $2 \mathrm{G}$ ).

The outcome measures at baseline were correlated with CMTNSv2, disease duration and the physical component of the SF-36v2 (SF-36v2PC). The results are listed in the online supplementary table 1 . Outcome measures that moderately/ strongly correlated with all three correlation variables include 
A

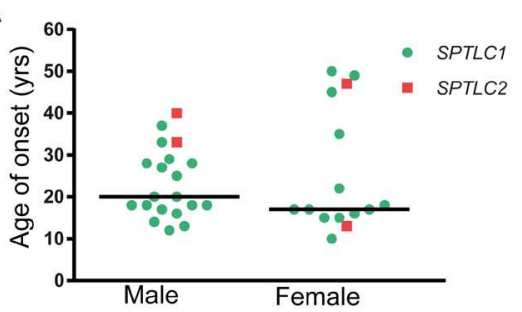

B

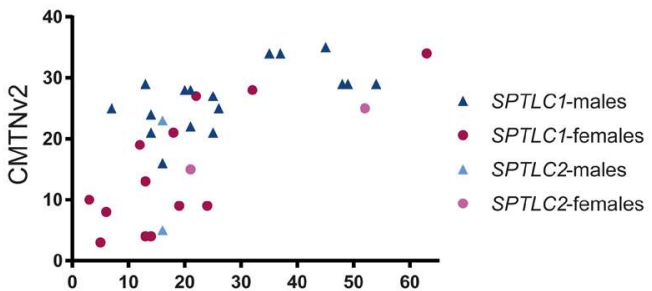

F

G
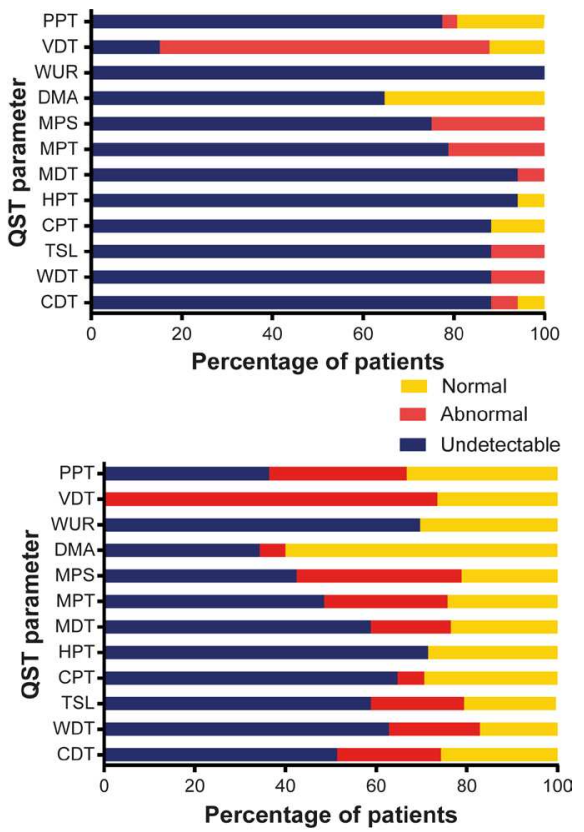

H

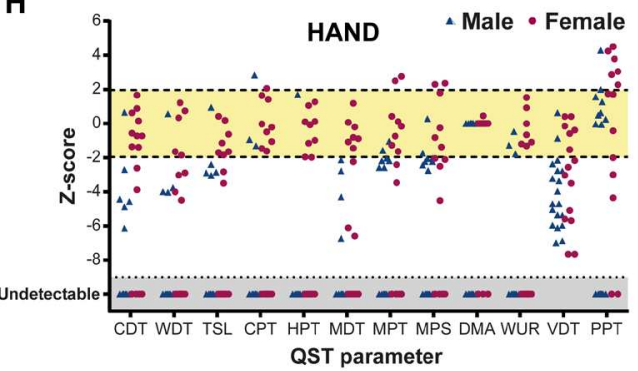

I

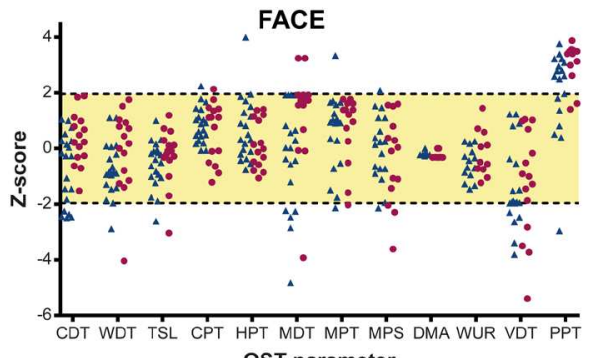

QST parameter

J

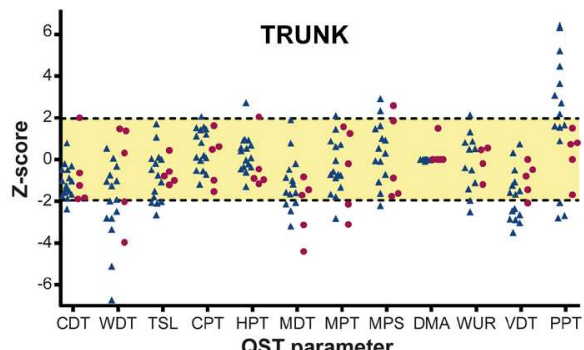

Figure 1 Patient demographics and baseline cross-sectional data (part 1). (A) Variation in age of onset between male and female patients with SPTLC1 and SPTLC2 mutations. (B) Disease progression in male and female SPTLC1 and SPTLC2 patients. (C) Tukey box plot showing the distribution of CMTNSv2 in males and females. There is a significant difference in disease severity between males and females ( ${ }^{*} \mathrm{p}=002$, Mann-Whitney test). Box: IQR; horizontal line: median; whiskers: 1.5 IQR; blue triangle: major outlier in the male group. (D, E) Large floor effects are seen in the sensory (D) and motor (E) nerve conduction studies in the upper limbs in both males and females. $(F, G)$ Quantitative sensory testing (QST) in feet (F) and hands (G). Most of the responses are undetectable/beyond the recordable range. $(\mathrm{H}-\mathrm{J})$ Somatosensory profiles determined with QST in the hands $(\mathrm{H})$, face $(\mathrm{I})$ and trunk (J). Data are expressed as Z-scores with SDs. Yellow area indicates normal range $(95 \% \mathrm{CI})$. Z values below ' 0 ' indicate loss of function and above ' 0 ' indicate gain of function. CDT, cold detection threshold; CMAP, compound muscle action potential; CMTNSv2, Charcot-Marie-Tooth Neuropathy Score version 2; CPT, cold pain threshold; DMA, dynamic mechanical allodynia; HPT, heat pain threshold; MDT, mechanical detection threshold; MPS, mechanical pain sensitivity; MPT, mechanical pain threshold; PPT, pressure pain threshold; SNAP, sensory nerve action potential; TSL, thermal sensory limen; VDT, vibration detection threshold; WDT, warm detection threshold; WUR, wind-up ratio. 
Table 2 Baseline neurophysiology (nerve conduction studies and quantitative sensory testing)

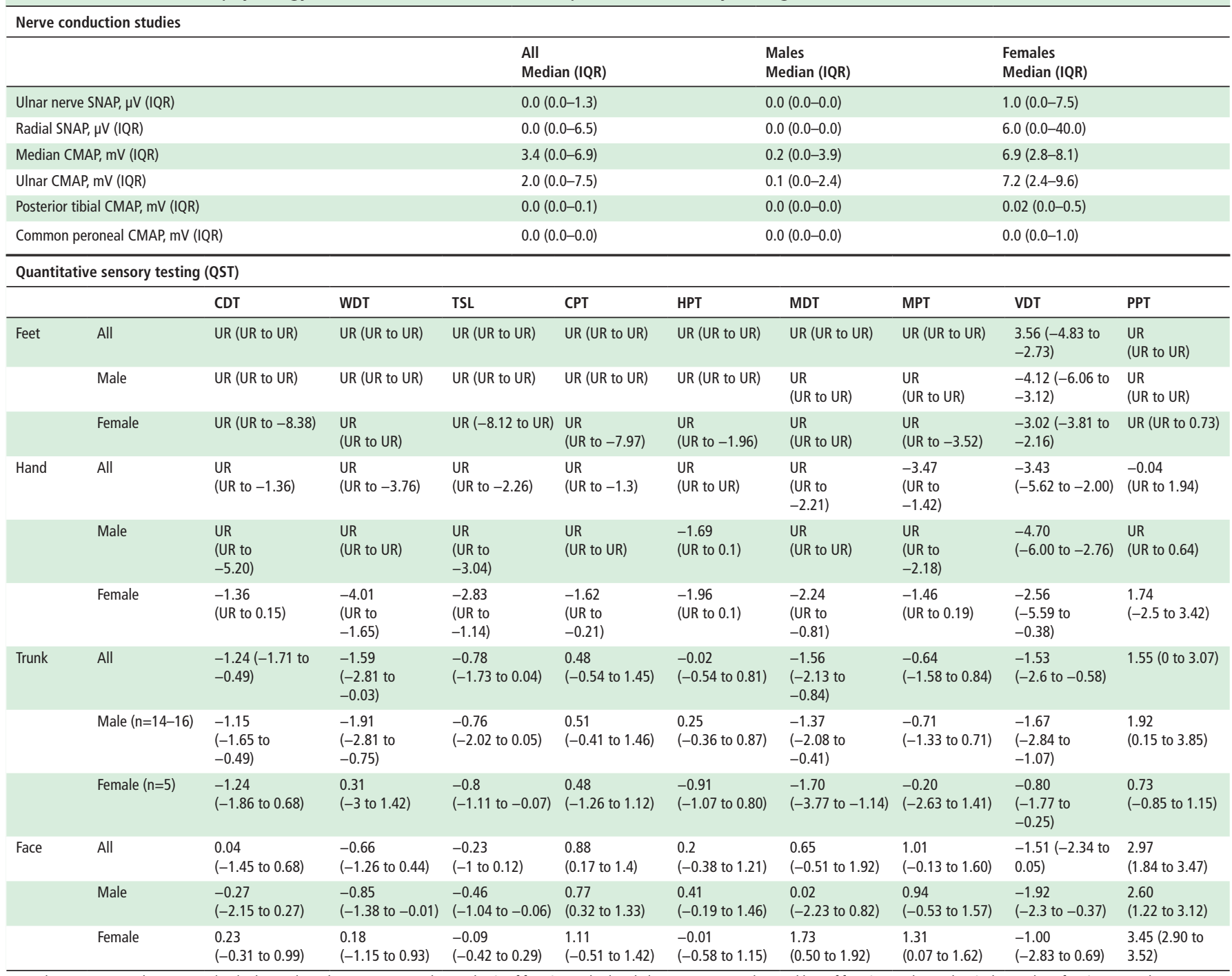

QST data are expressed as Z-scores (IQR) where values above 2 represent abnormal gain of function and values below -2 represent abnormal loss of function. In the trunk, $\mathrm{n}$ is the number of patients tested. CDT, cold detection threshold; CMAP, compound muscle action potential; CPT, cold pain threshold; HPT, heat pain threshold; LL, lower limb; MDT, mechanical detection threshold; MPT, mechanical pain threshold; PPT, pressure pain threshold; SNAP, sensory nerve action potential; TSL, thermal sensory limen; UR, unrecordable; VDT, vibration detection threshold; WDT, warm detection threshold.

NCS, QST-Hand, isometric ankle dorsiflexion/plantar flexion/ inversion/eversion and calf muscle fat fraction.

\section{Change over 12 months}

Three patients were lost to follow-up (online supplementary figure 1). Follow-up assessments were done after 1 year (mean $=364$ days \pm 7 days). Detailed results are summarised in tables 4 and 5. There was no significant change in the CMTNSv2, CMTNSv2-R, NCS, IENFD, NPSI or SF-36v2 over 12 months. Plasma 1-deoxysphinganine levels significantly decreased over 1 year (mean change $-0.13 \mu \mathrm{M}, 95 \%$ CI -0.24 to -0.02 , $\mathrm{p}=0.02)$.

In QST, significant changes reflecting clinical deterioration were only seen in Hand-PPT $(p=0.04)$, Face-VDT $(p=0.04)$ and Face-PPT $(p<0.0001)$. Significant deterioration was noted in ankle plantar flexion $(\mathrm{p}=0.0007)$, ankle inversion $(\mathrm{p}=0.03)$ and ankle eversion $(\mathrm{p}=0.006)$ (table 5$)$.

For MRI, 25 patients with HSN and 10 controls were analysed at 12 months (table 5 and figure $2 \mathrm{H}$ ). Other than the three patients lost to follow-up, other data are 'missing completely at random' due to technical reasons with the MRI or data transfer. There were significant increases in all the individual calf muscle fat fractions and the overall calf muscle fat fraction ( $p$ values range $=0.03$ to $<0.0001$ ) whereas there was no significant change in the control group ( $p$ values range $=0.16-0.79$ ).

In summary, calf muscle fat fraction, Hand-PPT and isometric ankle plantar flexion, inversion and eversion significantly changed over 12 months and correlated with CMTNSv2, SF36-v2PC and disease duration. Their relative responsiveness (SRMs) are shown in table 6. Calf muscle fat fraction had the best responsiveness both in individual muscles and as overall calf muscle fat fraction (overall calf muscle fat fraction SRM 0.81 vs SRM of 0.49 for Hand-PPT and SRM $<0.40$ for myometry). Overall calf muscle fat fraction changes were smaller in patients with baseline fat fraction less than $5 \%$ or greater than $70 \%$ (figure $2 \mathrm{H}$ ). All patients with overall calf muscle fat fraction less than $5 \%$ had CMTNSv2 of 10 or less. Patients with fat fraction greater than $70 \%$ had CMTNSv2 ranging from 28 to 34. Focusing on the patients with baseline overall calf muscle fat fraction of 5\%-70\% (20 patients, 59\% of MRI cohort) markedly improved the responsiveness (overall calf muscle fat fraction SRM of 1.60). 
Table 3 Baseline measures for computerised myometry, intraepidermal nerve fibre density, plasma deoxysphingolipid levels, SF-36v2 scores and calf muscle fat fraction

\begin{tabular}{|c|c|c|c|}
\hline & $\begin{array}{l}\text { All } \\
\text { Median (IQR) }\end{array}$ & $\begin{array}{l}\text { Males } \\
\text { Median (IQR) }\end{array}$ & $\begin{array}{l}\text { Females } \\
\text { Median (IQR) }\end{array}$ \\
\hline \multicolumn{4}{|l|}{ Myometric measurements } \\
\hline Isometric ankle dorsiflexion (Nm) & $0(0-18)$ & $0(0-3)$ & $10(0-23)$ \\
\hline Isometric ankle plantar flexion (Nm) & $7(0-25)$ & $2(0-18)$ & $20(1-27)$ \\
\hline Isometric ankle inversion (Nm) & $5(0-14)$ & $4(0-14)$ & $5(2-14)$ \\
\hline Isometric ankle eversion (Nm) & $4(0-12)$ & $2(0-9)$ & $6(1-14)$ \\
\hline Isometric knee extension (Nm) & $100(63-137)$ & $132(66-152)$ & $86(63-100)$ \\
\hline Isometric knee flexion (Nm) & $51(32-71)$ & $64(31-76)$ & $45(32-55)$ \\
\hline \multicolumn{4}{|l|}{ Intraepidermal nerve fibre density (IENFD) } \\
\hline IENFD (fibres/mm) & $0.1(0.0-1.8)$ & $0.0(0.0-1.3)$ & $1.2(0.1-2.7)$ \\
\hline \multicolumn{4}{|l|}{ Plasma deoxysphingolipids } \\
\hline 1-Deoxysphinganine $(\mu \mathrm{M})$ & $0.79(0.49-1.03)$ & $0.94(0.50-1.23)$ & $0.54(0.32-0.99)$ \\
\hline 1-Deoxysphingosine $(\mu \mathrm{M})$ & $1.5(0.96-2.40)$ & $1.68(1.18-2.48)$ & $1.13(0.80-2.18)$ \\
\hline \multicolumn{4}{|l|}{ Neuropathic Pain Symptom Inventory (NPSI) } \\
\hline Total NPSI score (0-100) & $25(14-37)$ & $23.5(15-40)$ & $22.0(13-36)$ \\
\hline Paroxysmal subscore (0-10) & $5(3-8)$ & $6(3-8)$ & $5(2-7)$ \\
\hline \multicolumn{4}{|l|}{ SF-36v2 } \\
\hline Physical component score & $37.5(33.5-49.8)$ & $34.8(31.9-40.2)$ & $51.4(36.6-55.9)$ \\
\hline Mental component score & $53.6(44.8-59.3)$ & $51.4(43.9-58.6)$ & $54.3(46.4-59.9)$ \\
\hline Physical functioning & $34.6(26.9-53.7)$ & $30.8(54.0-41.3)$ & $44.2(32.7-57.5)$ \\
\hline Role-physical & $47.1(34.1-57.2)$ & $39.2(32.5-51.5)$ & $57.2(45.9-57.2)$ \\
\hline Bodily pain & $42.2(38.2-50.7)$ & $43.0(8.1)$ & $45.0(12.3)$ \\
\hline Calf muscle fat fraction & & & \\
\hline
\end{tabular}

\begin{tabular}{|c|c|c|c|c|c|c|c|c|c|}
\hline & $\begin{array}{l}\text { All } \\
\text { HSN1 group } \\
\text { Median FF\% (IQR) }\end{array}$ & $\begin{array}{l}\text { All Control group } \\
\text { Median FF\% (IQR) }\end{array}$ & $P$ value & $\begin{array}{l}\text { HSN1 Males } \\
\text { Median FF\% } \\
\text { (IQR) }\end{array}$ & $\begin{array}{l}\text { Control Males } \\
\text { Median FF\% (IQR) }\end{array}$ & $P$ value & $\begin{array}{l}\text { HSN1 Females } \\
\text { Median FF\% } \\
\text { (IQR) }\end{array}$ & $\begin{array}{l}\text { Control Females } \\
\text { Median FF\% } \\
\text { (IQR) }\end{array}$ & $P$ value \\
\hline Tibialis anterior & $13.1(2.9-41.9)$ & $0.8(0.7-1.8)$ & $<0.0001$ & $14.3(4.9-41.1)$ & $0.9(0.7-2.1)$ & 0.0002 & $12.0(1.4-44.3)$ & $0.8(0.7-1.3)$ & 0.02 \\
\hline Extensor hallucis longus & $16.8(3.3-57.3)$ & $1.3(1.0-1.9)$ & $<0.0001$ & $17.8(10.6-59.8)$ & $1.9(1.1-3.4)$ & 0.0009 & $16.0(1.6-54.7)$ & $1.2(1.0-1.4)$ & 0.0003 \\
\hline Peroneus longus & $26.2(4.8-64.3)$ & $1.6(1.2-2.2)$ & $<0.0001$ & $37.0(18.3-69.3)$ & $2.2(1.7-4.4)$ & 0.0002 & $9.9(2.6-60.5)$ & $1.2(1.1-1.7)$ & 0.0004 \\
\hline Soleus & $24.4(4.8-63.2)$ & $1.6(1.1-2.6)$ & $<0.0001$ & $28.0(11.4-75.6)$ & $2.0(1.4-6.9)$ & 0.001 & $5.7(2.0-56.6)$ & $1.3(1.1-1.8)$ & 0.003 \\
\hline Lateral gastrocnemius & $37.1(4.5-79.8)$ & $1.2(1.2-2.4)$ & $<0.0001$ & $32.4(11.5-78.1)$ & $1.2(1.1-4.0)$ & 0.0006 & $49.7(2.0-82.5)$ & $1.3(1.1-2.1)$ & 0.005 \\
\hline Medial gastrocnemius & $33.9(4.2-71.8)$ & $1.4(1.0-2.8)$ & $<0.0001$ & $34.3(14.0-79.1)$ & $1.7(1.3-6.4)$ & 0.0003 & $3.0(1.3-52.5)$ & $1.0(0.9-1.7)$ & 0.02 \\
\hline Deep posterior group & $13.6(2.5-52.4)$ & $1.3(1.1-1.6)$ & $<0.0001$ & $15.7(9.2-54.0)$ & $1.6(1.1-3.1)$ & 0.0002 & $1.8(1.6-36.3)$ & $1.2(1.1-1.3)$ & 0.0005 \\
\hline Combined overall & $22.3(5.9-59.6)$ & $1.3(1.1-1.9)$ & $<0.0001$ & $22.7(9.6-66.6)$ & $1.5(1.3-5.3)$ & 0.0002 & $14.7(1.6-58.7)$ & $1.1(1.1-1.5)$ & 0.001 \\
\hline
\end{tabular}

Baseline measures are given as medians (IQR).

FF\%, fat fraction percentage; HSN1, hereditary sensory neuropathy type 1; SF-36v2, 36-Short Form Health Survey version 2 (1998).

\section{DISCUSSION}

In this study we describe the baseline characteristics and 12-month follow-up of a cohort of patients with HSN1 with SPTLC1 and SPTLC2 gene mutations. This is the first study to analyse intramuscular fat accumulation as a potential outcome measure in patients with HSN1.

In our baseline analysis, this study highlights the large phenotypical heterogeneity in SPTLC1 patients with HSN1 in the UK harbouring the same mutation (C133W). This heterogeneity was largely due to the differences in severity between males and females, where males were generally more severely affected. The significant difference in clinical severity, determined with the CMTNSv2, between males and females cannot be attributed to differences in plasma deoxysphingolipid levels which were not significantly different between the two sexes. Within the SPTLC1 female subgroup, there appeared to be phenotypic spectrum with females who have age of onset and disease progression similar to males and females with late onset and much slower rate of progression.

Dissociated sensory loss where nociception and temperature sensation (small fibre involvement) are affected early and more severely compared with vibration and proprioception (large fibre involvement) is well documented in HSN1. ${ }^{12}$ QST profiles demonstrate that there is also early large fibre involvement with deficits in mechanical perception mirroring that of temperature perception. We noted, as previously reported, ${ }^{27}$ an initial period of hypersensitivity to noxious pressure (lowered PPTs) and hyperalgesia (increased MPS). Despite the name, prominent motor involvement was seen in both male and female patients. We had previously used the same computerised myometry protocol that was used in this study to assess plantar flexion and dorsiflexion strengths in patients with CMT1A with ages similar to that of our cohort of patients with HSN1. ${ }^{13}$ On comparing the two groups, the patients with HSN1 had much greater motor impairment: mean isometric plantar flexion was $26.0 \mathrm{Nm}$ $(\mathrm{SD}=14.3)$ and dorsiflexion was $10.8 \mathrm{Nm}(\mathrm{SD}=7.5)$ in patients with CMT1A versus median isometric plantar flexion of $7 \mathrm{Nm}$ $(\mathrm{IQR}=0-25)$ and dorsiflexion of $0 \mathrm{Nm}(\mathrm{IQR}=0-18)$ in patients with HSN1.

For our longitudinal study we used a comprehensive range of measures including clinical, neurophysiological, myometric, plasma 1-deoxySL levels, IENFD and MRI measurement of calf intramuscular fat accumulation. To determine the validity of the outcome measures, the measures were correlated with a clinical 
A

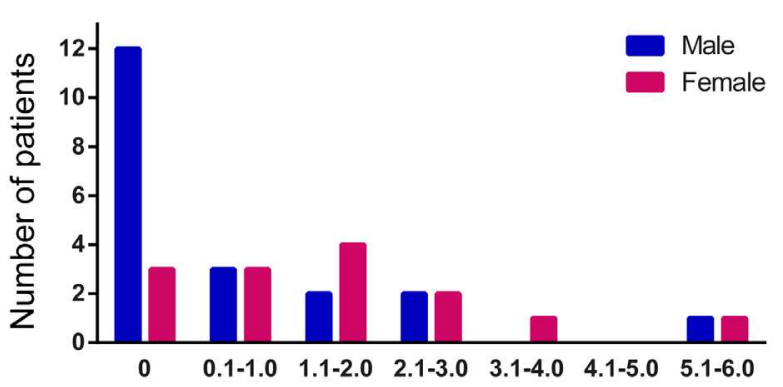

Intraepidermal nerve fibre density (fibres $/ \mathrm{mm}$ )

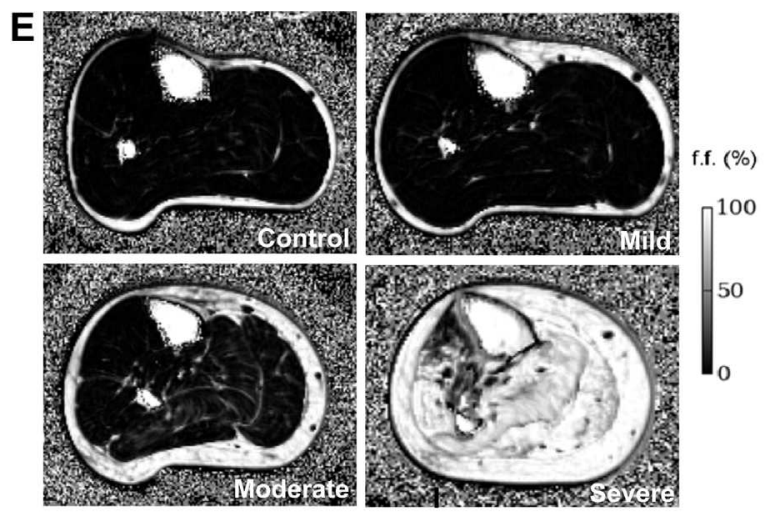

B

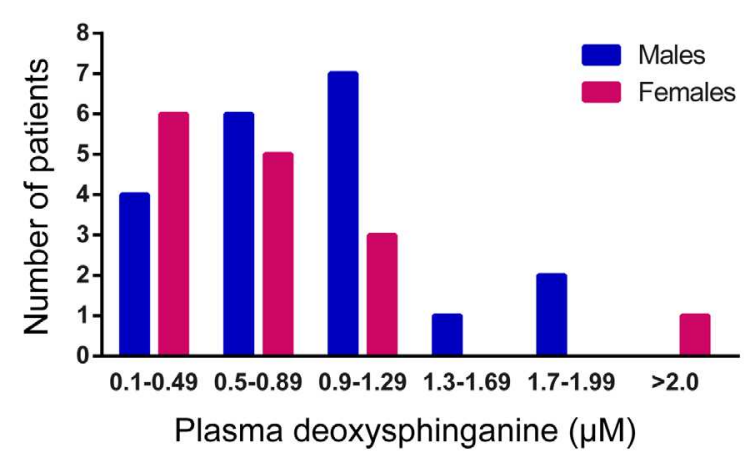

C

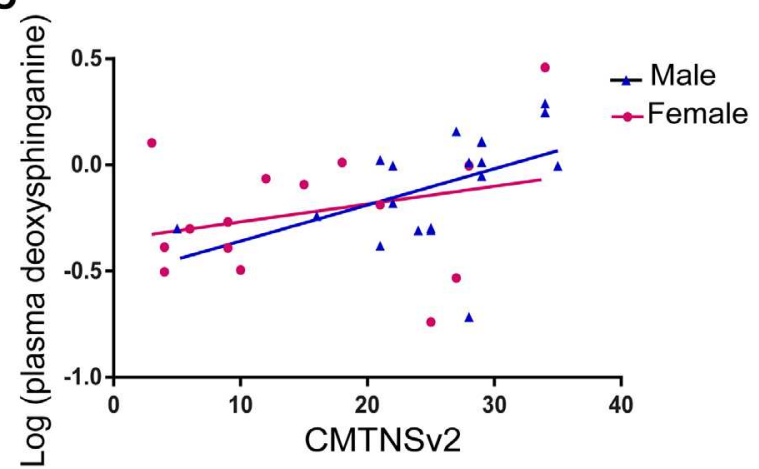

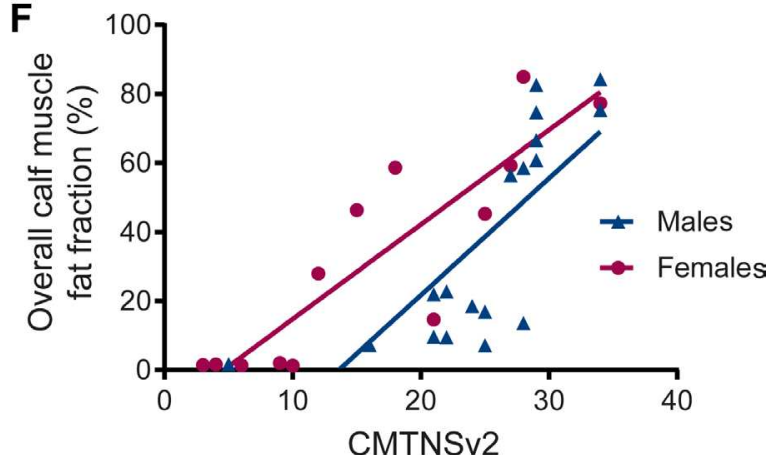

G

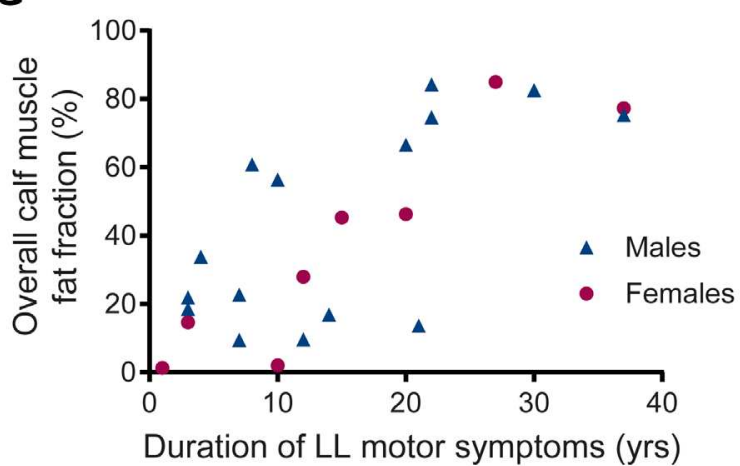

D

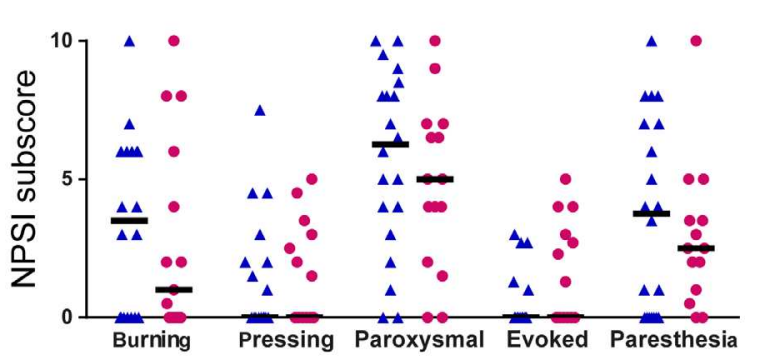

$\triangle$ Male

- Female

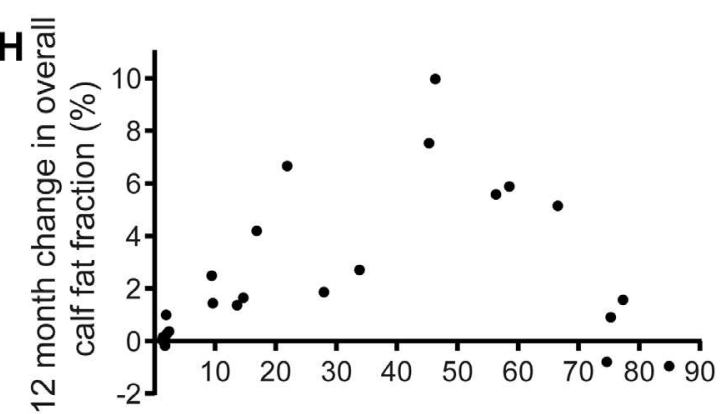

Baseline overall calf fat fraction (\%)

Figure 2 Baseline cross-sectional data (part 2) and MRI characteristics. (A) Distribution of intraepidermal nerve fibre density (IENFD) measures in males and females. (B) Distribution of plasma 1-deoxysphinganine levels in males and females. (C) Correlation between CMTNSv2 and plasma 1-deoxysphinganine. (D) Neuropathic Pain Symptom Inventory (NPSI) profiles in males and females. Solid bar: median. (E) Fat fraction map of a control male subject, mildly affected (CMTNSV2=5), moderately affected (CMTNSv2=16) and severely affected (CMTNSV2=34) male patients with hereditary sensory neuropathy type 1 (HSN1). (F) Correlation between overall calf muscle fat fraction and CMTNSv2. (G) Change in overall calf muscle fat fraction with duration of lower limb (LL) motor symptoms in males and females. $(\mathrm{H})$ Degree of change in overall calf muscle fraction over 1 year depends on its baseline value. CMTNSv2, CharcotMarie-Tooth Neuropathy Score version 2. 


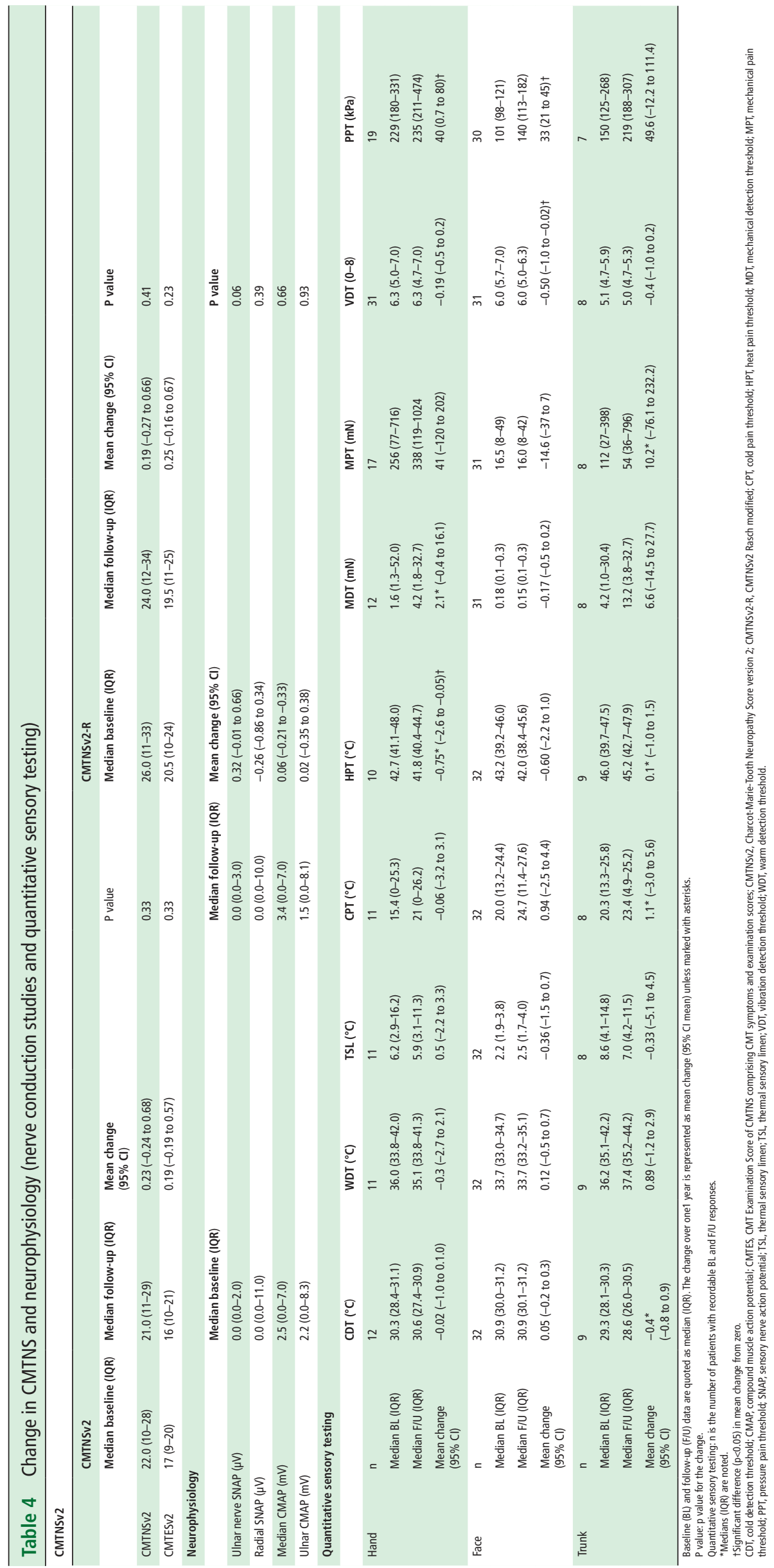




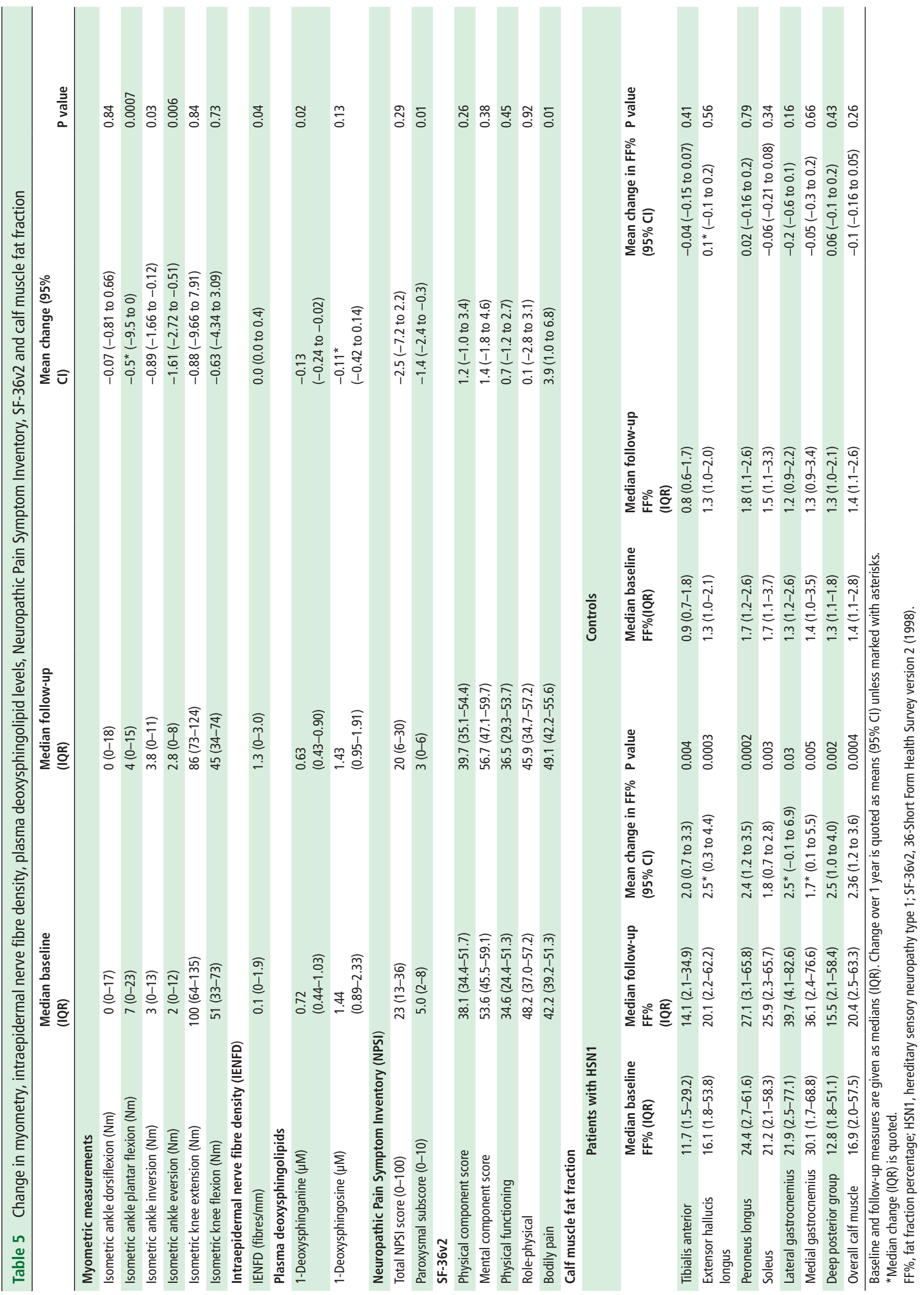


Table 6 Responsiveness of tests which had a significant change over 12 months

\begin{tabular}{|c|c|c|c|}
\hline & Mean change $(95 \% \mathrm{Cl})$ & SRM & $P$ value \\
\hline \multicolumn{4}{|l|}{ Quantitative sensory testing } \\
\hline Hand-PPT (kPa) & $40(0.7$ to 80$)$ & 0.49 & 0.046 \\
\hline \multicolumn{4}{|l|}{ Computerised myometry } \\
\hline Isometric ankle plantar flexion (Nm) & $-0.50^{*}(-9.5$ to 0$)$ & na & 0.0007 \\
\hline Isometric ankle inversion ( $\mathrm{Nm})$ & $-0.89(-1.66$ to -0.12$)$ & -0.33 & 0.03 \\
\hline Isometric ankle eversion (Nm) & $-1.61(-2.72$ to -0.51$)$ & -0.40 & 0.006 \\
\hline Extensor hallucis longus (FF\%) & 2.93 (1.50 to 4.37$)$ & 0.84 & 0.0003 \\
\hline Peroneus longus (FF\%) & 2.35 (1.22 to 3.48$)$ & 0.86 & 0.0002 \\
\hline Soleus (FF\%) & 1.75 (0.66 to 2.84 ) & 0.66 & 0.003 \\
\hline Lateral gastrocnemius (FF\%) & $2.46^{*}(-0.12$ to 6.87$)$ & na & 0.003 \\
\hline Medial gastrocnemius (FF\%) & $1.68^{*}(0.10$ to 5.49$)$ & na & $<0.0001$ \\
\hline Extensor hallucis longus (FF\%) & 4.94 (2.70 to 7.19$)$ & 1.33 & 0.0004 \\
\hline Peroneus longus (FF\%) & 4.25 (3.01 to 5.48 ) & 2.07 & $<0.0001$ \\
\hline Soleus (FF\%) & 3.33 (1.62 to 5.04$)$ & 1.18 & 0.001 \\
\hline Lateral gastrocnemius (FF\%) & $4.66(-0.56$ to 9.89$)$ & 0.54 & 0.08 \\
\hline Medial gastrocnemius (FF\%) & $6.57(2.35$ to 10.79$)$ & 0.94 & 0.005 \\
\hline Deep posterior group (FF\%) & 4.91 (3.01 to 6.80$)$ & 1.56 & 0.0001 \\
\hline Overall calf muscle (FF\%) & 4.34 (2.70 to 5.99$)$ & 1.60 & $<0.0001$ \\
\hline
\end{tabular}

Change is reported as mean change unless marked with an asterisk.

${ }^{*}$ Median change (IQR) is reported.

FF\%, fat fraction percentage; HSN1, hereditary sensory neuropathy type 1; PPT, pressure pain threshold; SRM, standardised response mean; na, not applicable as SRM values cannot be calculated in cases where the change is not normally distributed.

composite measure of disability, CMTNSv2, a patient self-assessment score, SF36-v2PC and disease duration. Calf muscle fat fraction, NCS, QST in the hand and isometric ankle plantar flexion, dorsiflexion, inversion and eversion correlated strongly/ moderately with all three validation variables.

Due to the heterogeneity in the cohort, many of the outcome measures were limited by both floor and ceiling effects. No significant changes were observed for most of the measures over 12 months and hence cannot be used in an HSN1 clinical trial if the aim of the therapy is disease stabilisation. These include CMTNSv2, CMTNSv2-R, NCS, IENFD, NPSI and SF-36v2.

A recent randomised, placebo-controlled trial in patients with HSN using oral L-serine supplementation by Fridman et $a l^{7}$ did not show a significant difference in the primary outcome (proportion of patients progressing more than one point on the CMTNS at 1 year) between L-serine and placebo groups. However, it showed a decline in CMTNS ( -1.5 units, $95 \%$ CI -2.8 to 0.1 , $\mathrm{p}=0.03)$ in $\mathrm{L}$-serine participants relative to placebo whereas the placebo group experienced a mean increase in CMTNS of 1.1 point $( \pm 0.53, p=0.04)$. The difference in the CMTNS progression in their placebo group and our study (change in CMTNS $=0.23,95 \% \mathrm{CI}-0.24$ to 0.68 ), despite having patients with similar distribution of severity (mean CMTNS $=24.6$ [7.0] vs median $=22.0$ [10-28]), could be secondary to differences in sample size. The annual change in CMTNS noted in patients with CMT1A ranged from -0.92 to +1.0 unit. $^{8-11} 28$ This variability highlights the limitations of using the CMTNS as a primary outcome measure in slowly progressive neuropathies.

Comparable changes over 1 year were noted in NCS and SF36v2 between this study and the placebo group of the randomised trial. ${ }^{7}$ Greater within-group variability was seen for IENFD (thigh) in the trial. This could reflect differences in techniques used for analysis.

In the current study of the neurophysiological measures, only Hand-PPT significantly changed over 12 months and correlated strongly with all three validation variables. However, it only has small/moderate responsiveness $(\mathrm{SRM}=0.49)$ and it is unlikely that QST parameters could be used singly as a primary outcome measure.

Significant change over 1 year was noted in myometric measurements of ankle plantar flexion, ankle inversion and ankle eversion; however, ankle inversion and eversion have small responsiveness (SRMs <0.40). SRM could not be calculated for ankle plantar flexion as the change was not normally distributed but if normal distribution was assumed, it would have moderate responsiveness with an SRM of 0.62 (mean $=-4.5 \mathrm{Nm}, 95 \% \mathrm{CI}$ -7.2 to $-1.9 \mathrm{Nm}$ ). Isometric ankle plantar flexion could therefore be explored as a potential secondary outcome measure in HSN1.

Surprisingly, a small but significant decrease in the plasma 1-deoxysphinganine level was found after 1 year. Differences in diet (intake of serine and alanine) and technical factors (differences in number of freeze-thaw cycles, transport time from UK to Switzerland) between the two time points could be possible explanations. Plasma 1-deoxySL levels have been shown to dramatically decrease with L-serine treatment and remain suppressed for the duration of treatment. ${ }^{67}$ Therefore, plasma 1-deoxySL levels are potentially very useful to show compliance and target engagement in an L-serine trial aiming at reducing these levels but they are unlikely to be useful as a marker of 
disease stabilisation. It is possible that in the context of neuropathy, 1-deoxySL levels in cerebrospinal fluid may be more relevant; however, no studies have investigated this in patients with HSN1 .

MRI determined fat quantification using the Dixon method has been shown to be reliable, sensitive and responsive as an outcome measure in recent natural history studies in various neuromuscular disorders. ${ }^{132930}$ Of all the tests performed, the MRI fat fraction quantification is the most promising as an outcome measure in HSN1. Despite large heterogeneity in the HSN1 group, the changes in both combined overall fat fraction and individual muscle fat fraction over 12 months were significant in patients with HSN1 whereas healthy controls showed no significant change. It also showed greater responsiveness (overall calf muscle fat fraction SRM 0.81) when compared with the other outcome measures in the study (SRM <0.49). In a previous study we reported a comparable change/responsiveness in overall calf fat fraction (SRM 0.83) in a 12-month follow-up study in a cohort of patients with CMT1A. ${ }^{13}$

Assessments focusing on sensory impairment (sensory NCS, most of the QST parameters and IENFD) have been hampered by floor effects. Due to the variable episodic nature of the neuropathic pain, another element of the condition, assessing longitudinal change will be difficult, especially with many of the existing pain questionnaires capturing only short periods of time. Despite the name HSN1, we have shown that there is significant motor involvement with a consistent pattern of disease progression: early progressive sensory involvement followed later by progressive motor involvement. The purpose of this study was to assess which of the potential outcome measures showed the largest effect size over 12 months across the whole cohort. This was calf muscle fat fraction. To translate this into responsiveness in a clinical trial, the intervention must be able to exert an effect on the outcome measure in question; which would be expected for intramuscular fat fraction unless the intervention only affected sensory pathways. This should be borne in mind when designing clinical trials, an intervention which did not slow the progression of intramuscular fat accumulation could not be considered a complete treatment, and we have shown that it is the most responsive measure of motor axonal loss.

The effect size can be increased further if we select patients with baseline overall calf muscle fat fraction of 5\%-70\% (20 patients, 59\% of MRI cohort), which doubles the responsiveness of overall calf muscle fat fraction (from SRM of 0.81 to 1.60). With this responsiveness, the number required in each study arm to detect a $50 \%$ reduction in disease progression is 25 . This is a feasible number to be recruited for a clinical trial in the UK.

The minimum clinically significant difference ${ }^{31}$ in fat fraction is yet to be determined. It remains important to include overall disease severity scores such as the CMTNSv2 and patient-reported outcome measures in a clinical trial to confirm the longitudinal validity of change in MRI calf muscle fat fraction. We have assessed this over 4 years in CMT1A and initial analysis shows promising results. ${ }^{32}$ Further analysis of a more proximal slice in those patients with HSN1 with $>70 \% \mathrm{FF}$ at the analysed level and a more distal slice for those with $<5 \% \mathrm{FF}$ may result in higher responsiveness of MRI muscle fat fraction over the full range of disease severity.

In conclusion, this study highlights the marked clinical heterogeneity of patients with HSN1 which is mainly due to sex. Calf muscle fat fraction, Hand-PPT and computerised myometric assessments of ankle plantar flexion and ankle inversion/eversion significantly changed over 1 year and correlated with disease severity, patient-reported health-related quality of life and disease duration. MRI determined calf muscle fat fraction is highly responsive and looks very promising as outcome measure to be included in therapeutic trials in HSN1.

\section{Author affiliations}

${ }^{1}$ MRC Centre for Neuromuscular Diseases, UCL Institute of Neurology and National Hospital for Neurology and Neurosurgery, London, UK

${ }^{2}$ Neuroradiological Academic Unit, UCL Institute of Neurology, London, UK ${ }^{3}$ Institute of Clinical Chemistry, University Hospital Zurich, Zurich, Switzerland ${ }^{4}$ Division of Neuropathology, National Hospital for Neurology and Neurosurgery, London, UK

${ }^{5}$ Fondazione I.R.C.C.S, Istituto Neurologico Carlo Besta, Milan, Italy

${ }^{6}$ Department of Biomedical and Clinical Sciences "Luigi Sacco", University of Milan, Milan, Italy

${ }^{7}$ Neurogenetics Unit, National Hospital for Neurology and Neurosurgery, London, UK ${ }^{8}$ Nuffield Department of Clinical Neurosciences, University of Oxford, Oxford, UK ${ }^{9}$ Department of Clinical Neurophysiology, Norfolk and NorwichUniversity Hospital, Norwich, UK

Acknowledgements We acknowledge Dr Maggie Williams and Dr Ruth NewburyEcob from Bristol Genetics Laboratory who helped with the recruitment of patients with HSN1. We also thank Professor Martin Koltzenburg and the Department of Clinical Neurophysiology at the National Hospital for Neurology and Neurosurgery, London, for the use of the QST equipment.

Contributors UK, JMM, CDJS, JST, TAY, DLHB, ML and MMR contributed to study concept and design. UK, MRBE, TH, SS, KO-A, GL, RL and JMP contributed to data acquisition and analysis. All authors contributed to drafting or revising the manuscript and figures.

Funding UK is grateful for fellowships from the National Institutes of Neurological Diseases and Stroke and Office of Rare Diseases (U54NS065712) and the Medical Research Council (519779). TH received support from the Swiss National Foundation SNF (Project 31003A_153390/1); Rare Disease Initiative Zurich ('radiz', Clinical Research Priority Program for Rare Diseases, University of Zurich). DLHB is a senior Wellcome clinical scientist (Ref 202747/Z/16/Z). MMR is grateful to the Medical Research Council (MRC), MRC Centre Grant (G0601943) and the National Institutes for Neurological Diseases and Stroke and Office of Rare Diseases (U54NS065712) for their support. This research was also supported by the National Institute for Health Research University College London Hospitals Biomedical Research Centre (BRC51/NS/MR).

Competing interests None declared.

\section{Patient consent for publication Not required.}

Ethics approval The study was approved by the local ethics committee.

Provenance and peer review Not commissioned; externally peer reviewed.

\section{REFERENCES}

1 Houlden H, King R, Blake J, et al. Clinical, pathological and genetic characterization of hereditary sensory and autonomic neuropathy type 1 (HSAN I). Brain 2006;129:411-25.

2 Auer-Grumbach M, De Jonghe P, Verhoeven K, et al. Autosomal dominant inherited neuropathies with prominent sensory loss and Mutilations. Arch Neurol 2003;60:329-34

3 Davidson GL, Murphy SM, Polke JM, et al. Frequency of mutations in the genes associated with hereditary sensory and autonomic neuropathy in a UK cohort. $J$ Neurol 2012;259:1673-85.

4 Penno A, Reilly MM, Houlden $\mathrm{H}$, et al. Hereditary sensory neuropathy type 1 is caused by the accumulation of two neurotoxic sphingolipids. J. Biol. Chem. 2010;285:11178-87.

5 Eichler FS, Hornemann T, McCampbell A, et al. Overexpression of the wild-type SPT1 subunit lowers desoxysphingolipid levels and rescues the phenotype of HSAN1. Journal of Neuroscience 2009;29:14646-51.

6 Garofalo K, Penno A, Schmidt BP, et al. Oral L -serine supplementation reduces production of neurotoxic deoxysphingolipids in mice and humans with hereditary sensory autonomic neuropathy type 1. J. Clin. Invest 2011:14-16.

7 Fridman V, Suriyanarayanan S, Novak $\mathrm{P}$, et al. Randomized trial of L-serine in patients with hereditary sensory and autonomic neuropathy type 1. Neurology 2019:92:e359-70. epub.

8 Lewis RA, McDermott MP, Herrmann DN, et al. High-dosage ascorbic acid treatment in Charcot-Marie-Tooth disease type 1A: results of a randomized, double-masked, controlled trial. JAMA Neurol 2013;70:981-7.

9 Micallef J, Attarian S, Dubourg 0, et al. Effect of ascorbic acid in patients with Charcot-Marie-Tooth disease type 1A: a multicentre, randomised, double-blind, placebo-controlled trial. Lancet Neurol 2009;8:1103-10. 
10 Pareyson D, Reilly MM, Schenone A, et al. Ascorbic acid in Charcot-Marie-Tooth disease type 1A (CMT-TRIAAL and CMT-TRAUK): a double-blind randomised trial. Lancet Neurol 2011;10:320-8.

11 Verhamme C, de Haan RJ, Vermeulen M, et al. Oral high dose ascorbic acid treatment for one year in young CMT1A patients: a randomised, double-blind, placebocontrolled Phase II trial. BMC Med 2009;7.

12 Fledrich R, Mannil M, Leha A, et al. Biomarkers predict outcome in Charcot-MarieTooth disease 1a. J Neurol Neurosurg Psychiatry 2017;88:941-52.

13 Morrow JM, Sinclair CDJ, Fischmann A, et al. MRI biomarker assessment of neuromuscular disease progression: a prospective observational cohort study. Lancet Neurol 2016;15:65-77.

14 Murphy SM, Herrmann DN, McDermott MP, et al. Reliability of the CMT neuropathy score (second version) in Charcot-Marie-Tooth disease. J Peripher Nerv Syst 2011;16:191-8.

15 Sadjadi R, Reilly MM, Shy ME, et al. Psychometrics evaluation of Charcot-Marie-Tooth neuropathy score (CMTNSv2) second version, using Rasch analysis. J Peripher Nerv Syst 2014;19:192-6.

16 Preston D, Shapiro B. Electromyography and neuromuscular disorders. Second. Philadelphia: : Elsevier, 2005.

17 Rolke R, Magerl W, Campbell KA, et al. Quantitative sensory testing: a comprehensive protocol for clinical trials. Eur J Pain 2006;10:77-88.

18 Pfau DB, Krumova EK, Treede R-D, et al. Quantitative sensory testing in the German Research Network on neuropathic pain (DFNS): reference data for the trunk and application in patients with chronic postherpetic neuralgia. Pain 2014;155:1002-15.

19 Lauria G. Skin biopsy in the diagnosis of peripheral neuropathies. Pract Neurology 2005;5:92-9.

20 Mwinyi J, Boström A, Fehrer I, et al. Plasma 1-deoxysphingolipids are early predictors of incident type 2 diabetes mellitus. PLoS One 2017:12:1-12.
21 Brazier $E$, Harper R, Jones NMB, et al. Validating the SF-36 health survey questionnaire: new outcome. Br MedJ 1992;305:160-4.

22 Maruish M, ed. User's manual for the SF-36v2 Health Survey. 3rd ed. Lincoln, RI,QualityMetrid Incorporated, 2011.

23 Glover GH, Schneider E. Three-point Dixon technique for true water/ fat decomposition withB0 inhomogeneity correction. Magn Reson Med 1991;18:371-83.

24 García de Yébenes Prous MJ, Salvanés FR, Ortells LC. Responsiveness of outcome measures. Reumatol Clínica 2008:4:240-7.

25 Piscosquito G, Reilly MM, Schenone A, et al. Responsiveness of clinical outcome measures in Charcot-Marie-Tooth disease. Eur J Neurol 2015;22:1556-63.

26 McArthur JC, Stocks EA, Hauer P, et al. Epidermal nerve fiber density. Arch Neurol 1998:55.

27 Rotthier A, Baets J, Timmerman V, et al. Mechanisms of disease in hereditary sensory and autonomic neuropathies. Nat Rev Neurol 2012:8:73-85.

28 Shy ME, Blake J, Krajewski K, et al. Reliability and validity of the CMT neuropathy score as a measure of disability. Neurology 2005:64:1209-14.

29 Bonati U, Hafner P, Schädelin S, et al. Quantitative muscle MRI: a powerful surrogate outcome measure in Duchenne muscular dystrophy. Neuromuscul Disord 2015;25:679-85

30 Willis TA, Hollingsworth KG, Coombs A, et al. Quantitative muscle MRI as an assessment tool for monitoring disease progression in LGMD2l: a multicentre longitudinal study. PLOS ONE 2013;8:e70993-12.

31 Copay AG, Subach BR, Glassman SD, et al. Understanding the minimum clinically important difference: a review of concepts and methods. Spine J 2007;7:541-6.

32 Evans M, Morrow J, Wastling S, et al. Proceedings-2017 Peripheral Nerve Society Meeting: MRI quantification of intramuscular fat accumulation in CMT1A: four year follow up data. Journal of the Peripheral Nervous System;22. 